Bull. Austral. Math. Soc.

$13 \mathrm{C} 13$

VOL. $64(2001)$ [21-26]

\title{
SEMI-BAER MODULES OVER DOMAINS
}

\author{
SANG Bum LeE
}

\begin{abstract}
For a commutative domain $R$ with 1 , an $R$-module $M$ is called a semi-Baer module if $\operatorname{Ext}_{R}^{1}(M, D)=0$ for all divisible $R$-modules $D$. We show that finitely generated modules of projective dimension at most 1 are semi-Baer modules and if $R$ is Prüfer or Matlis, then all modules of projective dimension at most 1 are semi-Baer modules.
\end{abstract}

In his seminal paper [1] on mixed Abelian groups, Baer proved that a countable Abelian group $B$ had to be free if $\operatorname{Ext}_{Z}^{1}(B, T)=0$ for all torsion Abelian groups $T$. The problem of determining those uncountable groups $B$ with this property turned out to be extremely difficult. Only 30 years later was it settled by Griffith [7] who showed that $B$ had to be free, no matter what its cardinality was.

The problem of characterising Baer modules $B$ over arbitrary domains $R$ (that is, $R$-modules $B$ with $\operatorname{Ext}_{R}^{1}(B, T)=0$ for all torsion $R$-modules $T$ ) was raised by Kaplansky [8]. He established two lemmas which led Eklof and Fuchs [2] to show that Baer modules over valuation domains likewise had to be free. With a lemma in that paper which dealt with regular cardinals as well as a version of Shelah's compactness theorem, Eklof, Fuchs and Shelah [3] proved a reduction theorem which reduces the problem of Baer modules to countably generated modules. As an application of the theorem, they proved that a module over an $h$-local Prüfer domain is a Baer module exactly if it is projective. Very recently, Fuchs and Salce [6, p.568] eliminated $h$ localness from the hypothesis and proved that a module over a Prüfer domain is a Baer module if and only if it is projective.

In this note, we study a weaker form of Baer modules. An $R$-module $M$ is called a semi-Baer module if $\operatorname{Ext}_{R}^{1}(M, D)=0$ for all divisible $R$-modules $D$ ( $D$ is divisible if $r D=D$ for all $0 \neq r \in R$ ). It turns out that these modules can be characterised in the same way as Baer modules (see Theorem 1). We also obtain a characterisation of divisible modules which might lead to a new study of divisible modules (see Corollary 4).

Received 13th July, 2000

The paper was completed while the author was visiting Tulane University. He expresses his thanks to the Department of Mathematics of Tulane University for the hospitality and Professor Laszlo Fuchs for several discussions on the subject.

Copyright Clearance Centre, Inc. Serial-fee code: 0004-9727/01 \$A2.00+0.00. 
Throughout this note, $R$ is a commutative domain with 1 which is not a field. For unexplained terminology, we refer to Fuchs and Salce [5].

In view of Kaplansky [8], we have

LEMma 1. Semi-Baer modules have projective dimension at most 1.

This is one of the two key properties of Baer modules which played a major role in their characterisations. The other one, flatness, is no longer guaranteed for semi-Baer modules as is illustrated by the module $\partial$ (see Fuchs and Salce [5, p.124]). Note also that we can restrict ourselves to torsion modules of projective dimension 1 regarding their semi-Baer property; to see this consider an exact sequence $0 \rightarrow F \rightarrow M \rightarrow T \rightarrow 0$ where $F$ is a free submodule of $M$ of projective dimension 1. Then $T$ is a torsion module of projective dimension 1 and the induced exact sequence $\operatorname{Ext}_{R}^{1}(T, D) \rightarrow \operatorname{Ext}_{R}^{1}(M, D) \rightarrow$ $\operatorname{Ext}_{R}^{1}(F, D)=0$ for a divisible module $D$ establishes the claim.

Recall that a submodule $N$ of $M$ is called tight if the projective dimension of $N$ and the projective dimension of $M / N$ are both less than or equal to the projective dimension of $M$. As in the case of Baer modules, we have

LEMma 2. Tight submodules of semi-Baer modules are again semi-Baer modules.

ProOF: Let $N$ be a tight submodule of a semi-Baer module $M$. Consider the exact sequence $0 \rightarrow N \rightarrow M \rightarrow M / N \rightarrow 0$ and the induced exact sequence $0=$ $\operatorname{Ext}_{R}^{1}(M, D) \rightarrow \operatorname{Ext}_{R}^{1}(N, D) \rightarrow \operatorname{Ext}_{R}^{2}(M / N, D) \rightarrow \ldots$ where $D$ is a divisible module. Since the projective dimension of $M / N$ is at most 1 by Lemma $1, \operatorname{Ext}_{R}^{2}(M / N, D)=0$. Hence, $\operatorname{Ext}_{R}^{1}(N, D)=0$, that is, $N$ is a semi-Baer module.

It is shown in [3] that countably generated, flat modules have projective dimension at most 1 and are countably presented. More generally, it can be shown that any countably generated module of projective dimension at most 1 is countably presented. Hence we have

COROLlary 1. Countably generated semi-Baer modules are countably presented.

A submodule $N$ of $M$ is said to be a $D E P$-submodule (Divisible Extension Property) if, for each divisible module $D$, the map $\operatorname{Hom}_{R}(M, D) \rightarrow \operatorname{Hom}_{R}(N, D)$ induced by the inclusion $N \rightarrow M$ is surjective.

LEMMA 3. Let $N$ be a submodule of a semi-Baer module $M$. Then it is a $D E P$ submodule of $M$ exactly if $M / N$ is a semi-Baer module.

Proof: The induced exact sequence

$$
\operatorname{Hom}_{R}(M, D) \rightarrow \operatorname{Hom}_{R}(N, D) \rightarrow \operatorname{Ext}_{R}^{1}(M / N, D) \rightarrow \operatorname{Ext}_{R}^{1}(M, D)=0
$$

induced from the inclusion $N \rightarrow M$, where $D$ is a divisible module establishes the result. 
Corollary 2. DEP-submodules of semi-Baer modules are tight. They are therefore semi-Baer modules.

The proof of the following lemma which is a verbatim version of Baer modules is the same as that of [2, Lemma 9] with the only change of $T E P$-submodules into $D E P$. submodules. For a module $M$, we denote the cardinality of a minimal generating set of $M$ by gen $M$.

LEMMA 4. Let $\kappa$ be an uncountable regular cardinal and $M$ a module with gen $M=\kappa$. Suppose

$$
0=M_{0}<M_{1}<\cdots<M_{\mu}<\ldots(\mu<\kappa)
$$

is a continuous well-ordered ascending chain of submodules of $M$ such that
(a) $M=\bigcup_{\mu<\kappa} M_{\mu}$
(b) gen $M_{\mu}<\kappa$ for each $\mu<\kappa$
(c) $M_{\mu}$ is a semi-Baer module for each $\mu<\kappa$.

If the set

$$
E=\left\{\mu<\kappa \mid \text { there exists } \beta>\mu \text { such that } M_{\beta} / M_{\mu} \text { is not semi-Baer }\right\}
$$

is stationary in $\kappa$, then $M$ is not a semi-Baer module.

With the aid of Lemma 4, we can prove:

THEOREM 1. A module $M$ is a semi-Baer module if and only if there exists a well-ordered continuous ascending chain of submodules

$$
0=M_{0}<M_{1}<\ldots<M_{\mu}<\ldots<M_{\kappa}=M \quad(\mu<\kappa)
$$

for some ordinal $\kappa$ such that, for each $\mu<\kappa, M_{\mu+1} / M_{\mu}$ is a countably generated semi-Baer module.

Proof: The proof is basically the same as that of [3, Theorem 10 and Theorem A] except that we start from the set $N$ of all countably generated semi-Baer modules instead of countably generated Baer modules. They are still countably presented by Corollary 1 and thus fit the proof.

Now we consider the converse of Lemma 1. Recall that a domain $R$ with its field of quotients $Q$ is called a Matlis domain if the projective dimension of $Q$ equals 1 . For a characterisation of Matlis domains, see Lee [9].

Theorem 2. Let $R$ be a Prüfer or Matlis domain and $M$ an $R$-module. Then $M$ is a semi-Baer module exactly if the projective dimension of $M$ is at most 1 .

Proof: If $M$ is an $R$-module of projective dimension 1 over a Prüfer domain $R$, then $M$ is the union of a well-ordered continuous ascending chain of submodules 
$0=M_{0}<M_{1}<\cdots<M_{\mu}<\cdots<M_{\kappa}=M(\mu<\kappa)$ such that $M_{\mu+1} / M_{\mu}$ is finitely presented and cyclic for each $\mu<\kappa$. Hence each $M_{\mu+1} / M_{\mu}$ is cyclic of projective dimension at most 1 and thus is a semi-Baer module by [5, p.36]. Then again by [5, p.74], $M$ is also a semi-Baer module. Now suppose $R$ is a Matlis domain. Then every divisible module $D$ is $h$-divisible. The exact sequence $0 \rightarrow H \rightarrow E \rightarrow D \rightarrow 0$ where $E$ is an injective module induces an exact sequence $0=\operatorname{Ext}_{R}^{1}(M, E) \rightarrow \operatorname{Ext}_{R}^{1}(M, D) \rightarrow$ $\operatorname{Ext}_{R}^{2}(M, H)$. The last Ext is 0 since the projective dimension of $M$ is at most 1 , proving the result.

Semi-Baer modules are abundant. Baer modules are trivial examples. The divisible module $\partial$ and its tight submodules are semi-Baer modules. By the same argument as in the case of $\partial$, all simply presented modules are also semi-Baer modules (see Fuchs [4]). It is shown in $[5$, p.41] that all $R D$-projective modules are semi-Baer modules. Now we try to identify a new class of semi-Baer modules.

Lemma 5. If $M$ is a module of projective dimension 1 , then $\operatorname{Tor}_{1}^{R}(M, A)=0$ for all torsion-free modules $A$.

Proof: Consider a projective resolution $0 \rightarrow H \rightarrow F \rightarrow M \rightarrow 0$ of the module $M$ where $F$ is free and $H$ is projective. From the induced exact sequence $0 \rightarrow \operatorname{Hom}_{R}(M, E) \rightarrow \operatorname{Hom}_{R}(F, E) \rightarrow \operatorname{Hom}_{R}(H, E) \rightarrow 0$ where $E$ is an injective module, we derive that the injective dimension of $\operatorname{Hom}_{R}(M, E)$ is at most 1 since both $\operatorname{Hom}_{R}(F, E)$ and $\operatorname{Hom}_{R}(H, E)$ are injective. The isomorphism

$$
\operatorname{Ext}_{R}^{1}\left(Q, \operatorname{Hom}_{R}(M, E)\right) \cong \operatorname{Hom}_{R}\left(\operatorname{Tor}_{1}^{R}(Q, M), E\right)=0
$$

implies that $\operatorname{Hom}_{R}(M, E)$ is a cotorsion module by [5, p.243]. We obtain $0=$ $\operatorname{Ext}_{R}^{1}\left(A, \operatorname{Hom}_{R}(M, E)\right) \cong \operatorname{Hom}_{R}\left(\operatorname{Tor}_{1}^{R}(A, M), E\right)$. The choice of $E$ implies that $\operatorname{Tor}_{1}^{R}(M, A)=0$.

Let $K$ denote the $R$-module $Q / R$. We call a module $D K$-injective if $\operatorname{Ext}_{R}^{1}(K, D)$ $=0$. It is easy to show that $K$-injective modules are $h$-divisible. Note also that over Matlis domains, $K$-injectivity, $h$-divisibility and divisibility are equivalent.

The next lemma is an analog of the well-known theorem that a module $F$ is flat if and only if its character module $F^{b}=\operatorname{Hom}_{Z}(F, Q / Z)$ is injective if and only if $F^{b}$ is absolutely pure.

LEMMA 6. For a module $A$, the following are equivalent:

(a) $A$ is torsion-free;

(b) $\operatorname{Hom}_{R}(A, E)$ is $K$-injective for all injective modules $E$;

(c) $\operatorname{Hom}_{R}(A, E)$ is $h$-divisible for all injective modules $E$;

(d) $\operatorname{Hom}_{R}(A, E)$ is divisible for all injective modules $E$. 
Proof: (a) $\Rightarrow$ (b) Since $\operatorname{Tor}_{1}^{R}(K, A)=0$ for all torsion-free modules $A, 0=$ $\operatorname{Hom}_{R}\left(\operatorname{Tor}_{1}^{R}(K, A), E\right) \cong \operatorname{Ext}_{R}^{1}\left(K, \operatorname{Hom}_{R}(A, E)\right)$ for all injective modules $E$. This implies that $\operatorname{Hom}_{R}(A, E)$ is $K$-injective.

(b) $\Rightarrow$ (c) $\Rightarrow$ (d) are trivial.

(d) $\Rightarrow$ (a) Let $t A$ be the torsion submodule of $A$. The inclusion $t A \rightarrow A$ induces an $R D$-exact sequence $0 \rightarrow \operatorname{Hom}_{R}(A / t A, E) \rightarrow \operatorname{Hom}_{R}(A, E) \rightarrow \operatorname{Hom}_{R}(t A, E) \rightarrow 0$. Here $\operatorname{Hom}_{R}(t A, E)$ is reduced and is at the same time divisible as an epic image of the divisible module $\operatorname{Hom}_{R}(A, E)$. Thus it is 0 . The choice of $E$ implies that $t A$ is 0 , and $A$ is torsion-free.

Recall that a finitely generated module has a finite projective resolution if it has a long projective resolution with finitely generated projective modules.

Lemma 7 . For a module $M$ of projective dimension 1, the following are equivalent

(a) $M$ is finitely generated;

(b) $M$ is finitely presented;

(c) $M$ has a finite projective resolution.

Proof: We have only to show that $(a) \Rightarrow(b)$. Consider a projective resolution $0 \rightarrow H \rightarrow F \rightarrow M \rightarrow 0$ of $M$ where $F$ is finitely generated, free and $H$ is projective. Since $H$ is of finite rank, $H$ is a summand of a finitely generated, free module $F_{1}$. Hence $H$ is trivially finitely generated, and $M$ is finitely presented.

Note that a finitely presented module $M$ of projective dimension 2 has always a finite projective resolution. Now we prove our main result.

THEOREM 3. All finitely generated modules of projective dimension at most 1 are semi-Baer modules.

PRoOF: Let $M$ be a finitely generated module of projective dimension at most 1 and $D$ a divisible module. By Lemma 7, $M$ has a finite projective resolution and thus we obtain a natural isomorphism (see Rotman $[10$, p.257])

$$
\operatorname{Tor}_{1}^{R}\left(M, \operatorname{Hom}_{R}(D, E)\right) \cong \operatorname{Hom}_{R}\left(\operatorname{Ext}_{R}^{1}(M, D), E\right)
$$

where $E$ is an injective module. Since $\operatorname{Hom}_{R}(D, E)$ is torsion-free, the Tor is 0 by Lemma 5 and thus the Hom on the right hand side is 0 . The choice of $E$ implies that $\operatorname{Ext}_{R}^{1}(M, D)=0$, and $M$ is a semi-Baer module.

Corollary 3. Let $M$ be a module of projective dimension at most 1. If there is a well-ordered continuous ascending chain

$$
0=M_{0}<M_{1}<\cdots<M_{\mu}<\cdots<M_{\kappa}=M \quad(\mu<\kappa)
$$


of submodules of $M$ such that for each $\mu<\kappa, M_{\mu+1} / M_{\mu}$ is finitely generated and of projective dimension at most 1 , then $M$ is a semi-Baer module.

Note that a module $P$ is absolutely pure if and only if $\operatorname{Ext}_{R}^{1}(N, P)=0$ for all finitely presented modules $N$. All $h$-divisible modules $H$ satisfy $\operatorname{Ext}_{R}^{1}(M, H)=0$ for all modules $M$ of projective dimension at most 1 . The next corollary is an analog of these results.

COROLlaRY 4. The following conditions on a module $D$ are equivalent:

(a) $D$ is divisible;

(b) $\operatorname{Ext}_{R}^{1}(R / R r, D)=0$ for every $r \in R$;

(c) $\operatorname{Ext}_{R}^{1}(R / L, D)=0$ for every projective ideal $L$ of $R$;

(d) $\operatorname{Ext}_{R}^{1}(M, D)=0$ for every finitely generated module $M$ of projective dimension at most 1 .

Proof: See [5, p.36] and Theorem 3.

\section{ReferenCeS}

[1] R. Baer, 'The subgroups of the elements of finite order of an abelian group', Ann. Math. 37 (1936), 766-781.

[2] P.C. Eklof and L. Fuchs, 'Baer modules over valuation domains', Ann. Mat. Pura Appl. 150 (1988), 363-373.

[3] P.C. Eklof, L. Fuchs and S. Shelah, 'Baer modules over domains', Trans. Amer. Math. Soc. 322 (1990), 547-560.

[4] L. Fuchs, 'Simply presented torsion modules over valuation domains', in Abelian groups and modules, Lecture Notes Pure Appl. Math. 182 (Marcel Dekker, New York, 1996), pp. 23-44.

[5] L. Fuchs and L. Salce, Modules over valuation domains, Lecture Notes in Pure and Appl. Math. 97 (Marcel Dekker, New York, 1985).

[6] L. Fuchs and L. Salce, Modules over non-noetherian domains, Math. Surveys and Monographs 84 (Amer. Math. Soc., Providence R.I., 2001).

[7] P. Griffith, 'A solution to the splitting mixed problem of Baer', Trans. Amer. Math. Soc. 139 (1969), 261-269.

[8] I. Kaplansky, 'The splitting of modules over integral domains', Arch. Math. 13 (1962), 341-343.

[9] S.B. Lee, 'On divisible modules over domains', Arch. Math. 53 (1989), 259-262.

[10] J. Rotman, An introduction to homological algebra (Academic Press, New York, 1975).

Department of Mathematical Education

Sangmyung University

Seoul 110-743

Korea 